\title{
An improved White Patch method for image illumination estimation
}

\author{
Li Zhao ${ }^{1}$, Wei Ma ${ }^{1}$, Mengxia Tang ${ }^{2}$, Songnan Chen* ${ }^{* 1,2}$ \\ ${ }^{1}$ School of information engineering, Xinyang Agriculture and Forestry University \\ No.1 North Circular Road, Pingqiao District,xingyang,464000. \\ ${ }^{2}$ School of technology, Beijing Forestry University \\ No.35 Tsinghua East Road, Haidian District, Beijing, 10086. \\ China
}

Received: July 13, 2020. Revised: August 22, 2020. Accepted: September 18, 2020. Published: September 22, 2020.

\begin{abstract}
As one of the underlying pixel-based illumination estimation algorithms, the White Patch algorithm is an algorithm for calculating the global illumination RGB value of an image based on the specific assumption that the maximum reflected light on the scene is chromatic. The algorithm is harsh on the assumptions of scene illumination, and many images are difficult to satisfy this assumption constraint. In this paper, we propose an improved White Patch image illumination estimation method. Firstly, the image patch is extracted by using sliding window method, we then use the white patch algorithm to estimate the illumination color value of each patch, and finally the kernel density estimation is adopted to obtain the overall illumination color value of the image. The experimental results show that the improved White Patch images illumination estimation method proposed to this paper performs better on the illumination estimation of natural illumination scene images.
\end{abstract}

Keywords-Illumination estimation, White path, Slide window, Color value.

\section{INTRODUCTION}

$\mathrm{T}$ HE ability of the human visual system to distinguish the color of surface of an object in the case of changing the color of the scene illumination is called color constancy [1]. In the field of machine vision, because the illumination of the image capturing scene is uncontrollable, the captured image may have color distortion problems. How to make a computer have the same color congruence as human beings, that is, reducing the influence of scene illumination on the color of the image makes the processing of the image more precise, which is one of the urgent problems to be solved. Color constancy calculation is one of the mainstream methods to solve the influence of scene illumination on images.

In the field of object recognition, color invariance is an important research clue. Funt et al. [15] point that the object recognition can be regarded as a set of different color features, indicating that different objection can be recognized by different color indexes. They proposed a robust color descriptor, color histogram ratio, which calculates the ratio of RGB color channels of adjacent pixels; Murase and Nayar[16] suggested that the image should be explicitly sampled under all possible observation conditions by considering the changes of light and angle of view for objection recognition; Khan et al. [17] used shape and color features of object recognition, and proposed a multi-cue classification method based on the separation of shape and color cues, using color information on a top-down specific category of attention mapping to guide attention, and then further deployment Color attention map, which adjusts shape features by getting more features from areas in the image that may contain object instances.

In the field of image segmentation, the color information about images has attracted more and more attention to researchers. There are two main reasons: first, the information contained in color images is more than that in gray images; second, the color feature of image is an important feature to identify the same region in the image [18]. For all the unique colors that is close to perception, they can be combined with a uniform area that represents the object of the images, so that the image becomes more meaningful and easier to analyze. Tan et al. [19] proposed an image segmentation method based on histogram threshold fuzzy C-means (HTFCM), which uses the histogram threshold technique to obtain the center of the probable unified region and the number of the center by analyzing the global features of the image, and then use the fuzzy C-means method to improve the density of each region. The histogram threshold technique fuzzy C-means method does not require complex calculations and is relatively simple to implement.

In the field of dynamic image compression, color space plays an important role in color image processing and color vision applications. In compressing images, properties of the human visual system are used to remove image details that are not perceptible to the human eye, known as mental visual 
redundancy. The effect of color space properties on compression efficiency is presented by Kahu et al [20]. They investigate the applicability of various color spaces to image and video compression. First, a comprehensive review of existing color spaces is provided and these color spaces are categorized and their strengths and limitations are highlighted; second, a quantitative analysis and benchmarking of color space from the perspective of image and video compression algorithms are conducted to identify and evaluate key color space parameters for image and video compression algorithms. Image compression requires describing the visual content of an image of a suitable color space that reduces psycho visual redundancy, so choosing an appropriate color space is critical for image compression algorithms, often as the first step in the algorithm.

In summary, scene illumination estimation is crucial to subsequent image processing, and image data inevitably interleaves scene illumination with valid features .Currently there are two types of methods of color constancy calculation: illumination estimation and color descriptor extraction. Due to changes in the lighting in the scene, the color information about the image has also undergone subtle changes. In order to achieve the extraction of image color descriptors, many researchers have done a lot of work. Ballard and Swain [21] proposed to use color histograms as color descriptors to describe objects, but this method is still different to light source changes. To solve this problem, Funt and Finlayson [22] proposed a lighting invariant indexing method, which is geometrically variable in illumination. Finalyson et al. [23] combined the above two theories and proposed an indexing method that is not affected by changes in brightness or light source changes, however, all the above methods are in terms of highlight still different. Gevers and Smeulders [24] combined light source and illumination geometry to propose an invariant for reflectivity. This work was later extended to the differential structure of the image [25], which can be used for edge and corner detection with constant luminosity. In addition, Gevers and Stokman [17] found that the instability caused by the nonlinear transformation of the luminosity invariant hindered the practical application of luminosity invariance theory. Based on the error analysis, they proposed a robust luminosity invariant. Weijer et al. [26] proposed a descriptor that connects color descriptors and shape descriptors. This descriptor has been successful in many computer vision applications such as object recognition, image matching and image retrieval.

The illumination estimation is to estimate the illumination RGB value of the image scene by some calculation and restore the image color to the color of the standard illumination condition; the color descriptor extraction is to extract the feature descriptor that is robust to illumination in the image. Image colors constancy calculations are the basis of subsequent image processing analysis, as Barnard said: "If a computer has the same color constancy as the human visual system and provides stable color features, then many tricky computer vision problems can be solved."
Compared with the color descriptor extraction, illumination estimation can restore the global color of the image, and can extract various features more accurately, not just color descriptors, so it is widely used in the field of image processing. The illumination estimation methods are divided into three categories: the illumination estimation method based on the underlying pixels. According to the underlying pixel features of the image and some assumptions, the statistical characteristics in the three channels of the image pixels $\mathrm{R}, \mathrm{G}$, and $\mathrm{B}$ are directly taken as the global illumination color RGB value of the image; based on the learning illumination estimation method, the images in the image set and their corresponding standard illumination are used to obtain the corresponding model through training. Then, using the trained model to estimate the RGB value of the scene illumination color of the input image of the novel datasets; based on the fusion illumination estimation method, the method integrates the first two types of illumination estimation methods, and optimizes or fuses the two methods to estimate the RGB values of the scene illumination color of the input image.

Gijsenij et al. [2] pointed out that the estimation of illumination color is an ill-posed problem. The illumination estimation method based on the underlying pixels begins with the Retinex theory proposed by Landin and McCann based on the human visual physiology characteristics [3], which believes that in the image, the slight change of color is caused by the change of the illumination color in the shooting scene. The dramatic change in color is caused by the spectral reflectance change of the surface of the object being photographed in the scene; based on this theory, Land and McCann proposed the Gray World algorithm [4] and the White Patch [26] algorithm. The Gray World algorithm and the White Patch algorithm assume that the color values corresponding to the average reflectance and the maximum reflectance of the surface of the object being photographed in the scene are achromatic. These two algorithms are limited by the scene. For example, when the color in the image capturing scene is not rich enough, the Gray World algorithm has a poor illumination estimation effect; when there is no white surface in the image capturing scene, the White Patch algorithm has a poor illumination estimation effect [5].

In order to improve the applicability of Gray World algorithm, Xu et.al proposed two optimization methods, namely the weight-based and image entropy-based Gray World algorithm. The weight-based Gray World algorithm first divides the image to be processed into several small image blocks, and then calculates the average value and standard deviation of the three channels of each image block R, G, and B, finally calculates the global illumination color RGB value by weighting the standard deviation of each image block [6]. Since the Gray World algorithm is not ideal for estimating images with less color, image entropy is used to reduce its influence. Image entropy is a statistical form of information contained in the image, reflecting the average amount of information in the image. The Gray World algorithm based on image entropy first calculates the one-dimensional discrete relative entropy of the 
three color channels of the image, then calculates the gain coefficients of the three channels R, G, and B, and finally performs coefficient correction [6]; in order to make the Gray World algorithm and the White Patch algorithm is more universal, Finlayson and Trezzi introduced Minkowski norm into these two algorithms, and proposed the Shades of Gray algorithm [7]; Weijer et al. proposed the Gray Edge algorithm based on the assumption that the average reflectance difference in the scene is colorless, and introduced image edge information (lower and higher order) into the illumination estimation in the opposite color space [8].

The White Patch method, one of the very first color constancy methods, estimates the illuminant color from the maximum response of three color channels. White Patch algorithm has poor illumination estimation and generalization ability. Aim at this problem, in the paper we propose a simple optimization method for White Patch algorithm: firstly, use the sliding window method to extract small blocks with a step size of 1 pixel, and then use White Patch algorithm to estimate the light color of each small block, then The global illumination of the image is obtained using kernel density estimation.

\section{METHOD}

The White Patch algorithm is an underlying pixel-based illumination estimation algorithm based on the assumption that the maximum reflectance of a subject in a scene are achromatic [3]; according to this hypothesis, the White Patch algorithm calculates the pixels of three channels of RGB in the image. The maximum value is used to determine the scene illumination RGB value of the image, as shown in Equation 1.

$$
\left(l_{r}, l_{g}, l_{b}\right)=\left(\max \left(p_{r}\right), \max \left(p_{g}\right), \max \left(p_{b}\right)\right)
$$

Since the White Patch algorithm is based on the specific assumption that the maximum reflectance of the surface of the object being photographed in the scene are colorless, it is difficult to satisfy this assumption of many scene images, which results in poor performance and applicability of the algorithm in some scenarios. In this paper, an improved White Patch images illumination estimation method is proposed. Firstly, the image is divided into several small blocks, and then the white patch algorithm is used to estimate the illumination color of each small block. Then the kernel density is used to estimate the global illumination of the image. The flow is shown in Figure 1.

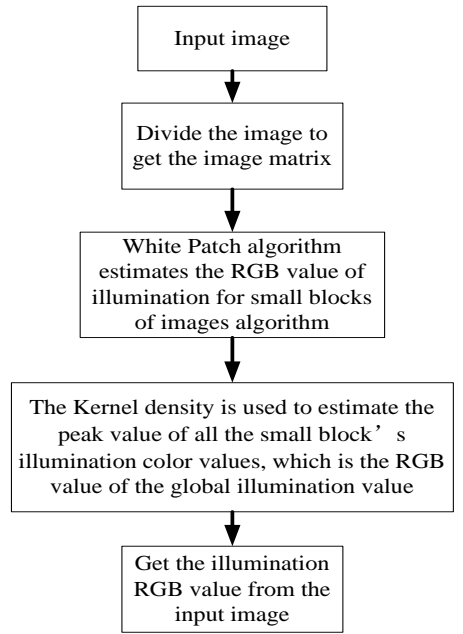

Fig.1 flow chart of improved White Patch image illumination estimation method.

\section{A. Image segmentation}

Under natural conditions, the illumination received by the image is non-uniform. After the image is segmented, the illumination information about the block can be better estimated according to the local color information of the image, thereby obtaining the overall illumination RGB value of the image. Assuming that the size of the image $I$ is $m \times n \times 3$, sliding the image of a window of $k \times k \times 3$, and a total of $m$ image patches can be obtained to form an image matrix $I_{p}$, as shown in Equation 2.

$$
\mathbf{I}_{p}=\left[\begin{array}{cccc}
I_{11} & I_{12} & \cdots & I_{1(n-k+1)} \\
I_{21} & I_{22} & \cdots & I_{2(n-k+1)} \\
\vdots & \vdots & \ddots & \vdots \\
I_{(m-k+1) 1} & I_{(m-k+1) 2} & \cdots & I_{(m-k+1)(n-k+1)}
\end{array}\right]
$$

\section{B. Estimation of illumination color}

After the image matrix $I_{p}$ is obtained, the White Patch algorithm is used to estimate the illumination RGB value of each small block, and the illumination color value $i l l u_{i}=\left(R_{i}, G_{i}, B_{i}\right)$ of the $i$-th small block can be obtained, and the $(m-k+1)(n-k+1)$--group illumination values are shared to form the illumination matrix $i l l u_{i}$ of the image $I$, as shown in equation (3).

$$
\begin{aligned}
& I L L U_{I}=\left[i l l u_{1}^{T}, i l l u_{2}^{T}, \ldots, i l l u_{(m-k+1)(n-k+1)}^{T}\right] \\
& =\left[\begin{array}{cccc}
R_{1} & R_{2} & \ldots & R_{(m-k+1)(n-k+1)} \\
G_{1} & G_{2} & \ldots & G_{(m-k+1)(n-k+1)} \\
B_{1} & B_{2} & \ldots & B_{(m-k+1)(n-k+1)}
\end{array}\right]
\end{aligned}
$$

We need to estimate the distribution of the $(m-k+1)(n-k+1)$-group illumination values in the illumination matrix illu $_{i}$. Kernel density estimation is a method used to estimate the unknown density function of probability 
theory [10]. Since each set of illumination values contain three independent illumination components, we use a three-dimensional kernel density estimation algorithm to estimate the distribution of illumination values. The illumination matrix $i l l u_{i}$ of the image $I$ is regarded as $\mathrm{m}$ independent and identically distributed illumination vectors $i l l u_{i}$, and each illumination vector contains three components, which are $(R, G, B)^{T}$. Supposing the probability density function is $f$, and then the kernel density is estimated as shown in Equation 4.

$f_{h}($ illu $)=\frac{\sum_{i-1}^{n} K_{h}\left(i l l u-i l l u_{i}\right)}{(m-k+1)(n-k+1) h}=\frac{\sum_{i=1}^{n} K\left(\frac{i l l u-i l l u_{i}}{h}\right)}{(m-k+1)(n-k+1) h}$

Where $x_{c}$ is the kernel function center, $\sigma$ is the width parameter of the function, bandwidth $\mathrm{h}>0$, $K_{h}(x)=\frac{1}{h} K\left(\frac{x}{h}\right)$ is a scaling kernel function, $K(\bullet)$ is a Gaussian kernel function, substitute it into formula 4 and get formula 5 .

$$
f_{h}(i l l u)=\frac{\sum_{i=1}^{n} \exp \left[\frac{-\left\|i l l u-i l l u_{i}\right\|^{2}}{(2 \sigma)^{2}}\right]}{(m-k+1)(n-k+1) h}
$$

The distribution of illumination vector $i l l u_{i}$ can be obtained by kernel density estimation, and the peak value of illumination vector distribution is selected as the RGB value of global illumination of the image.

\section{EXPERIMENTAL RESULTS AND ANALYSIS}

\section{A. Datasets and Evaluation}

In this paper, the Color Checker [11], [12] and SFU Lab Datasets [13] are used to evaluate the effectiveness of our method. The Color Checker datasets consists of 568 images from natural light conditions, including indoor and outdoor scenes. The SFU Lab datasets consists of 321 images from indoor light conditions, which is an image set of several same objects under different color of the light. We evaluate the effect of our method in different scenes and the experimental results show that our proposed approach is more accurate than competing methods.

In this paper, the angle error [14] is selected as the performance evaluation, which is the error between the reproduction of the real colorless surface under white light and the actual reproduction of the colorless surface when the estimated light source color is separated. As shown equation 6 and 7:

$$
E_{r e}=\cos ^{-1}\left(\frac{\overrightarrow{e_{a}} / \overrightarrow{e_{e}} \bullet U}{\left|\overrightarrow{e_{a}} / \overrightarrow{e_{e}}\right| \bullet \sqrt{3}}\right)
$$

$$
U \approx \frac{\overrightarrow{e_{a}}}{\overrightarrow{e_{a}}}(7)
$$

We selected the mean, median, maximum and minimum values of angle error as the evaluation indicator. The smaller value of the evaluation, the estimated illumination is closer to the standard and the corresponding algorithm is better.

\section{B. Sets the Size of Sliding Window}

In the first step, our method needs to use the sliding window to obtain the image block matrix, in which the size of the sliding window directly affects the performance of our method and needs to be determined through experiments. Figure 2 shows the relationship between window size and the angle error of our method. From figure 2(a) we can find the error value decreases as the window size increases until $\mathrm{k}=575$, so $\mathrm{k}=575$ is set as the best value on the Color Checker datasets. From figure 2(b) we also find the error value is least when $k=300$ on the SFU Lab datasets.

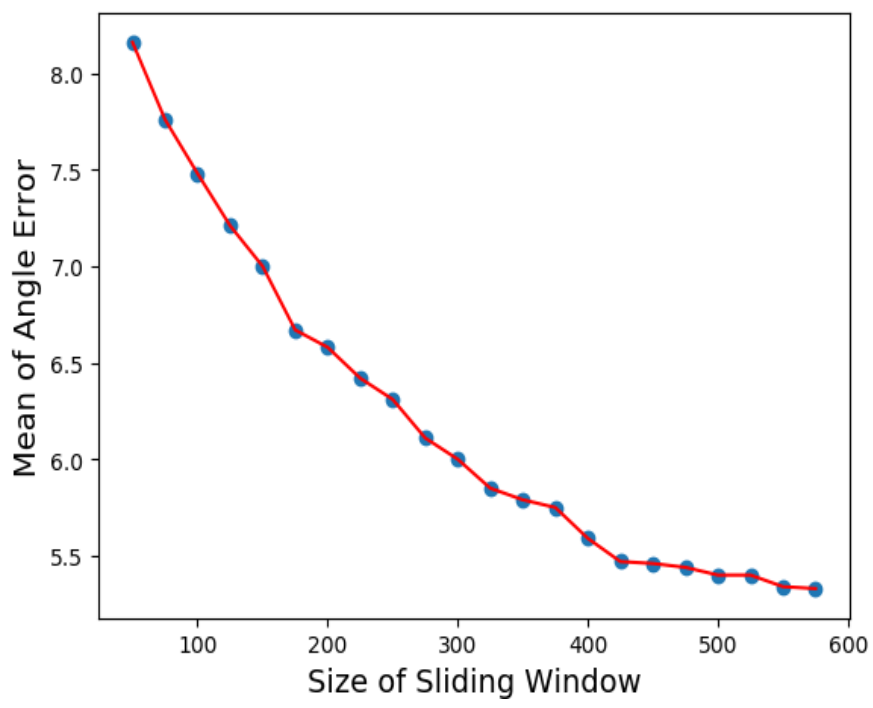

(a) Color Checker datasets

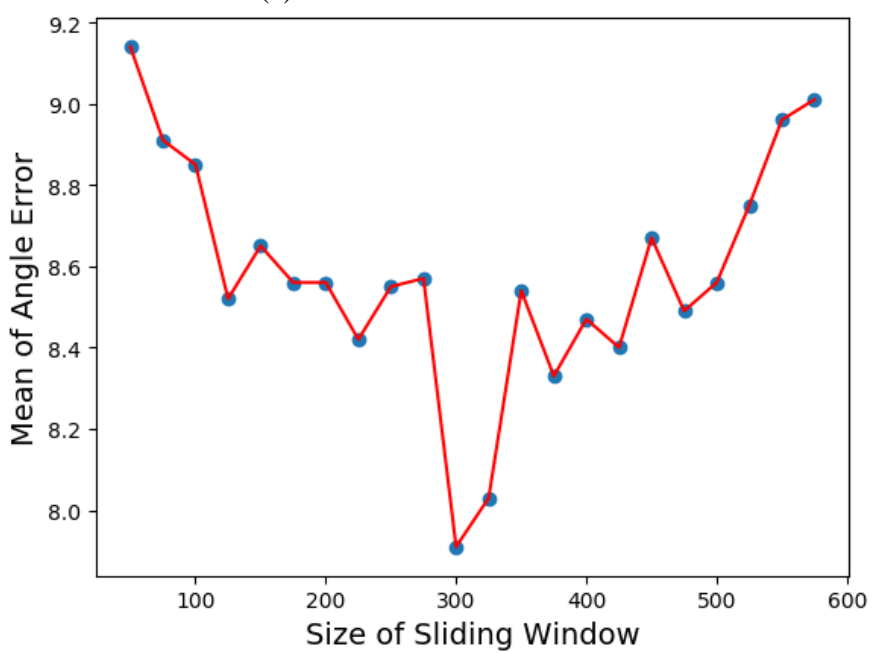

(b) SFU Lab datasets

Fig. 2 the relation between the size of slide window and the mean error of angle on Color Checker and SFU Lab datasets. 


\section{Analysis of Experimental Results}

In this section, we take angle error as the evaluation standard. We compare the proposed method with the traditional White Patch algorithm and other illumination estimation algorithms based on the low-level pixel. Table 1 shows the results on the Color Checker and SFU Lab datasets. We can find that compared with the traditional White Patch method, the mean error of our method is reduced by 7.03 degrees and the accuracy is improved by $60 \%$. Compared with other illumination estimation algorithms based on the low-level pixel, our method has smallest error, indicating that compared with the traditional algorithm or other similar algorithms, our method performs well; In the SFU Lab datasets, we can find that compared with the traditional White Patch algorithms, the mean error of our method is reduced by 1.81 degrees, the effect is not obvious. Compared with other illumination estimation algorithms based on low-level pixels, our method is only superior to Gray World and also has no obvious advantages. Therefore, the method proposed in this paper is not well in the SFU Lab datasets, but the effect in the Color Checker datasets is better, indicating that our method is more suitable for natural lighting scene.

Table 1. Baseline comparison on Color Checker and SFU Lab datasets. In this table, WP is White Patch method [27], GW is Gray World method [4], SOG is Shades of edge method [22], GE is Gray-Edge method [26], 1stGE is $1^{\text {st }}$ Gray-Edge method [26] and 2 stGE is $2^{\text {st }}$ Gray Edge method[26]. MEAN represents the mean of the angle error, MED is the median of the angle error, MAX is the maximum value of the angle error and MIN represents the minimum value of angle error. (unit: degree).

\begin{tabular}{|c|c|c|c|c|c|c|c|c|}
\hline \multirow{2}{*}{ Method } & \multicolumn{4}{|c|}{ Color Checker image set } & \multicolumn{4}{c|}{ SFU Lab image set } \\
\cline { 2 - 9 } & MEAN & MED & MAX & MIN & MEAN & MED & MAX & MIN \\
\hline GW & 8.74 & 7.79 & 30.32 & 0.06 & 10.09 & 7.49 & 36.96 & 0.04 \\
\hline SOE & 6.96 & 6.10 & 26.89 & 0.29 & 6.89 & 3.94 & 31.50 & 0.09 \\
\hline GE & 5.91 & $\mathbf{4 . 3 2}$ & 27.79 & 0.06 & 5.95 & 3.85 & 29.57 & 0.07 \\
\hline 1 st GE & 5.61 & 4.72 & 21.44 & 0.29 & 6.19 & 3.60 & 34.67 & 0.18 \\
\hline 2 stGE & 8.98 & 8.64 & 21.01 & 1.61 & 5.70 & 3.14 & 29.46 & 0.09 \\
\hline WP & 12.36 & 12.84 & 22.28 & 1.10 & 9.72 & 7.44 & 36.30 & 0.20 \\
\hline Ours & $\mathbf{5 . 3 3}$ & $\mathbf{4 . 4 5}$ & $\mathbf{2 4 . 0 8}$ & $\mathbf{0 . 0 9}$ & $\mathbf{7 . 9 1}$ & $\mathbf{6 . 1 1}$ & $\mathbf{3 0 . 4 7}$ & $\mathbf{0 . 2 5}$ \\
\hline
\end{tabular}

\section{CONCLUSION AND DisCUSSION}

As one of As one of the ways to achieve color constancy in machine vision, the illumination estimation algorithm mainly estimates the RGB value of the scene lighting color of the image, and then restores the overall color of the image to achieve the effect of shooting under white light, thus reducing the color shift of the image. White Patch is an illumination estimation algorithm based on low-level pixels, which is proposed to a specific assumption. Although limited by the scene, the algorithm is computed simply. In this paper, we propose an improved method of White Patch algorithm. Firstly, we use the sliding window method to extract the image block.
Then white patch algorithm is used to estimate the illumination value of each block. Finally, the RGB value of scene illumination is obtained by kernel estimator for the density function. The experimental results show that the method proposed to this paper can improve the accuracy of the traditional White Patch, especially in the natural lighting scene.

The research on the illumination estimation algorithm in this paper is limited to the assumption of a single light source. Most of the existing multi-light source illumination estimation algorithms are also based on a single, which divides the image into regions for single illumination estimation, and finally performs fusion. Therefore, the selections of single illumination as well as image region are crucial. A single illumination estimation algorithm with shorter time, better effect and simple calculation can further promote the comprehensive development of illumination estimation algorithms. In addition, the lack of image sets of multi-illumination scenes is one of the obstacles to the study of multi-illumination estimation algorithms. In future research as we gain more and more in-depth understanding of machine vision, we can further study other features or use the relationship model between these features and ground-truth to improve the accuracy of illumination estimation.

\section{ACKNOWLEDGMENT}

This work was supported in part by Key R\&D and Promotion Project of Henan Province (Grant No. 182102210131, 1821021110160); Government Decision and Research Bidding project of Henan Province (Grant No. 2018B145); Young Teachers Fund of Xinyang Agriculture and Forestry University (Grant No. 201701013).

\section{References}

[1]. X. Zh. Xu, L. Zhuo, J. Zhang, "Research on color constancy under open illumination conditions," INTERNATIONAL JOURNAL OF ELECTRONICS, vol. 26, no. 5, pp. 681-686, 2009.

[2]. A. Gijsenij, T. Gevers, J. V. D. Weijer, "Computational color constancy: survey and experiments," IEEE Transactions on Image Processing, vol. 20, no. 9, pp. 2475-2489, 2011.

[3]. K. T. Blackwell, G. Buchsbaum, "Quantitative studies of color constancy," Journal of the Optical Society of America A Optics \& Image Science, vol. 5, no. 10, pp. 1772-1780, 1988.

[4]. Land, H. Edwin, "The retinex theory of color vision," Scientific American, vol. 237, no. 6, pp. 108-128, 1978.

[5]. Zh. Tang, H. Zh. Liu, J. Zh. Yuan, "Advances Research on Color Constancy Computation under Single Illuminant," Computer Science, vol. 43, pp. 12-18, 2016

[6]. X. Zh. Xu, Y. H. Chai, X. M. Liu, "Improved Grey World Color Correction Algorithms," Acta Photonica Sinica, vol. 39, no. 3, pp. $559-564,2010$.

[7]. G. D. Finlayson, E. Trezzi, "Shades of gray and color constancy," in IS\&T/SID 12th Color Imaging Conf, 2004, pp. 37-41.

[8]. J. V. D. Weijer, T. Gevers, A. Gijsenij, "Edge-based color constancy," IEEE Transactions on Image Processing, vol. 16, no. 9, pp. 2207-2214, 2007.

[9]. B. Funt, K. Barnard, L. Martin, "Is machine colour constancy good enough?" in Proc. European Conf on Computer Vision, 1998, pp. 445-459.

[10]. M. Ester, H. P. Kriegel, J. Sander, "A density-based algorithm for discovering clusters in large spatial databases with noise," in Proc. the 2nd International Conf on Knowledge Discovery and Data Mining, 1996, pp. 226-231. 
[11]. A. D. Logvinenko, B. Funt, C. Godau, "Metamer Mismatching," IEEE Trans. on Image Processing, vol. 23, no. 1, pp. 34-43, 2014.

[12]. P. V. Gehler, C. Rother, A. Blake, T. P. Minka, T. Sharp, "Bayesian color constancy revisited," in Proc. IEEE Computer Society Conf on Computer Vision and Pattern Recognition, 2008, pp. 24-26.

[13]. K. Barnard, L. Martin, B. Funt, "A data set for color research," Color Research \& Application, vol. 27, no. 3, pp. 147-151, 2012.

[14]. G. D. Finlayson, R. Zakizadeh, A. Gijsenij, "The reproduction angular error for evaluating the performance of illuminant estimation algorithms," IEEE Transactions on Pattern Analysis and Machine Intelligence, vol. 39, no. 7, pp. 1482-1488, 2017.

[15]. B. Funt, G. Finlayson, "Color constant color indexing," IEEE Transactions on Pattern Analysis and Machine Intelligence, vol. 17, no. 5, pp. 522-529, 1995.

[16]. H. Murase, S. K. Nayar, "Learning and recognition of 3D objects from appearance," in proc. of IEEE Work shop on Qualitative Vision, 1993, pp. 39-50.

[17]. F. S. Khan, J. V. D. Weijer, M.Vanrell, "Top down color attention for objects from appearance," in proc. of IEEE Work shop on Qualitative Vision, 1993, pp.39-50.

[18]. H. D. Cheng, X. H. Jiang, Y. Sun, J. L. Wang, Color image segmentation: advances and prospects, Pattern Recognition, vol. 34, no. 12, pp. 2259-2281, 2001.

[19]. K. Tan, N. Isa, Color image segmentation using histogram threshold-fuzzy C-means hybrid approach, Pattern Recognitions, vol. 44, no.1, pp.1-15, 2011

[20]. S. Kahu, R. Raut, K. Bhurchandi, Review and evaluation of color spaces for image/video compression, Color Research and Application, vol. 44, no. 1 , pp. 8-33,2019. •

[21]. D. H. Ballard, "Ganeralizinng the hough tranfrom to detect arbitrary shapes," Pattern Recognition, vol. 13, no. 2, pp. 111-122, 1981. •

[22]. B. Funt, G. Finlayson, "Color constant color indexing," IEEE Transactions on Pattern Analysis and Machine Intelligence, vol. 17, no. 5, pp. 522-529, 1995.

[23]. G. Finlayson, B. Schiele, J. Crowley, "Comprehensive colour image normalization," in Proc. of the $5^{\text {th }}$ European Conf on Computer Vision, 1998, pp. 475-490.

[24]. T. Gevers, “Color-based $\square$ object $\square$ recognition," $\square$ IEEE $\square$ Transactions on $\square$ Pattern $\square$ Analysis $\square$ and $\square$ Machine $\square$ Intelligence, vol. 23, no. 12, pp. $1338-1350,2001$

[25]. T. Gevers, H. Stokman, $\square$ "Robust $\square$ histogram $\square$ construction from color $\square$ invariants $\square$ for $\square$ object $\square \square$ recognition," $\square$ IEEE $\square$ Transactinson $\square$ Pattern $\square$ Analysis $\square$ and $\square$ Machine $\square$ Intelligence, vol. 26, no. 1, pp. 113-118, Feb. 2004.

[26]. J. V. D. Weijer, C. Schmid, "Coloring local feature extraction," in Proc. $9^{\text {th }}$ European Conf on Computer Vision, Austria, 2006, pp.334-348.

[27]. J J. Mccann, S. P. Mckee, T. H. Taylor, "Quantitative studies in retinex theory a comparison between theoretical predictions and observer resonses to the color Mondrian experiments," Vision Research, vol .16, no. 5, pp. 445-458, Feb. 1976.

Li Zhao, MA.Eng in Computer Science, associate professor, main research areas include computer vision and cloud computing.

Wei Ma, MA.Eng in Computer Science, lecturer, main research areas include computer vision and internet of things.

Mengxia Tang, Master Student in forestry engineering, main research areas include computer vision and Artificial intelligence.

Songnan Chen in $\mathrm{PhD}$. Candidate in forestry engineering, lecturer, main research areas include computer vision and Artificial.

\section{Creative Commons Attribution License 4.0 (Attribution 4.0 International , CC BY 4.0)}

This article is published under the terms of the Creative Commons Attribution License 4.0 https://creativecommons.org/licenses/by/4.0/deed.en_US 